\title{
Trends over Time of Lung Function and Radiological Abnormalities in COVID-19 Pneumonia: A Prospective, Observational, Cohort Study
}

\author{
Pierachille Santus ${ }^{1,2}{ }^{-}$, Nicola Flor ${ }^{3}$, Marina Saad ${ }^{1,2}$, Stefano Pini ${ }^{1,2}$, Elisa Franceschi ${ }^{1,2}$, Andrea Airoldi ${ }^{2}$, \\ Paolo Gaboardi ${ }^{1,2}$, Sonia Ippolito ${ }^{3}$, Maurizio Rizzi ${ }^{2}$ and Dejan Radovanovic ${ }^{2, *}$ \\ 1 Department of Biomedical and Clinical Sciences (DIBIC), Università degli Studi di Milano, \\ 20157 Milano, Italy; pierachille.santus@unimi.it (P.S.); marina.saad@unimi.it (M.S.); \\ stefano.pini@unimi.it (S.P.); elisa.franceschi@unimi.it (E.F.); paolo.gaboardi@unimi.it (P.G.) \\ 2 Division of Respiratory Diseases, Ospedale Luigi Sacco, Polo Universitario, ASST Fatebenefratelli-Sacco, \\ 20157 Milano, Italy; andrea.airoldi@asst-fbf-sacco.it (A.A.); maurizio.rizzi@asst-fbf-sacco.it (M.R.) \\ 3 Division of Radiology, Ospedale Luigi Sacco, Polo Universitario, ASST Fatebenefratelli-Sacco, \\ Via G.B. Grassi 74, 20157 Milano, Italy; nicola.flor@asst-fbf-sacco.it (N.F.); sonia.ippolito@asst-fbf-sacco.it (S.I.) \\ * Correspondence: dejan.radovanovic@asst-fbf-sacco.it; Tel.: +39-02-3904-2321
}

\section{check for}

updates

Citation: Santus, P.; Flor, N.; Saad, M.; Pini, S.; Franceschi, E.; Airoldi, A.; Gaboardi, P.; Ippolito, S.; Rizzi, M.; Radovanovic, D. Trends over Time of Lung Function and Radiological Abnormalities in COVID-19 Pneumonia: A Prospective, Observational, Cohort Study. J. Clin. Med. 2021, 10, 1021. https:// doi.org/10.3390/jcm10051021

Academic Editor: Luca Bertolaccini

Received: 19 January 2021

Accepted: 21 February 2021

Published: 2 March 2021

Publisher's Note: MDPI stays neutral with regard to jurisdictional claims in published maps and institutional affiliations.

Copyright: (c) 2021 by the authors. Licensee MDPI, Basel, Switzerland. This article is an open access article distributed under the terms and conditions of the Creative Commons Attribution (CC BY) license (https:// creativecommons.org/licenses/by/ $4.0 /)$.

\begin{abstract}
Radiological and functional sequelae of Coronavirus Disease 2019 (COVID-19) pneumonia are still poorly understood. This was a prospective, observational, physiological, cohort study on consecutive adult patients with COVID-19 pneumonia admitted in April-May 2020 in the high dependency respiratory unit of L. Sacco University Hospital in Milan (Italy). During hospitalization, patients underwent chest computed tomography (CT), blood gas analysis, spirometry, and lung diffusion capacity for carbon monoxide (DLco), which were repeated 6 weeks post-discharge. Chest CTs were individually read by two expert radiologists, that calculated the total severity score (TSS). Twenty patients completed the study (mean age 58.2 years, $70 \%$ males). During the acute phase, mean DLco, alveolar volume (VA), and vital capacity (VC) were 56.0 (16.3), 64.8 (14.0), and 71.7 (16.9) \% predicted, respectively, and were inversely associated with $\mathrm{PaO}_{2} / \mathrm{FiO}_{2}$ ratio. Fifty percent of patients had a restrictive ventilatory pattern; mean TSS was 7.9 (4.0). At follow up, gas exchange parameters were normalized; consolidations persisted in $10 \%$ of cases, while DLco was $<80 \%$ predicted in $65 \%$ of patients and was independently predicted by $\log _{10}$ D-dimer at admission $(\beta-18.675$; 95\%CI, -28.373--9.076; $p=0.001$ ). In conclusion, functional abnormalities in COVID-19 pneumonia survivors can persist during follow up and are associated with the severity of the disease.
\end{abstract}

Keywords: COVID-19; lung function; lung diffusion; D-dimer; DLCO; spirometry; chest CT

\section{Introduction}

Since the initial outbreak in Wuhan (China), the Coronavirus Disease 2019 (COVID19) pandemic has caused more than $1,200,000$ deaths worldwide [1], leading to an unprecedented burden on healthcare systems with hundreds of thousands patients needing hospitalization [2]. The lung damage caused by COVID-19 is characterized by a variable spectrum of interstitial, organizing, and acute fibrinous organizing pneumonia [3], reflecting ground glass opacities, crazy paving patterns, and pulmonary consolidations at the chest computed tomography (CT) [4]. In addition, hypoxic respiratory failure can be worsened by endothelial dysfunction and a pro-thrombotic diathesis that sustain a worsening of ventilation/perfusion mismatch and are responsible for increased risk for adverse outcomes in patients with severe disease $[3,5,6]$. The radiological evolution of COVID-19 pneumonia during the acute phase of the disease has been extensively studied $[7,8]$, but the pathophysiological and clinical impact of potential pulmonary sequelae is still under investigation. Some line of evidence suggested that thirty days from discharge the CT abnormalities resolve almost completely in mild to moderate COVID-19 
forms [9]. Other Authors, however, reported that in patients with moderate, severe and critical disease, both ground glass opacities and septal thickening tend to persist up to three months post-discharge [8,10-14]. Accordingly, the few available studies conducted on recovering COVID-19 patients have shown that the main lung function complications are represented by reduced lung diffusion capacity for carbon monoxide (DLCO) and, more rarely, by impaired static and dynamic volumes $[10-13,15,16]$. These observations are in line with previous studies conducted in Severe Acute Respiratory Syndrome Coronavirus (SARS-CoV) $[17,18]$ and Middle East Respiratory Syndrome Coronavirus (MERS-CoV) survivors [19]. However, differently from what was observed in SARS survivors [18], in convalescent COVID-19 patients lung function parameters were scarcely associated with the extension and quality of CT abnormalities $[10,11]$. Indeed, so far, the lung function pattern observed during follow up did not find a satisfactory explanation $[10,15,16]$, the greatest limitation being the lack of previous lung functional assessments to be compared with $[10,15,16]$.

By prospectively assessing lung function, gas exchange, and chest CT findings both during the acute phase and during follow up, we aimed at investigating the pulmonary pathophysiology and the short-term complications of COVID-19 pneumonia and at exploring the possible relationship between the evolution of functional abnormalities, gas exchange and radiological sequelae.

\section{Materials and Methods}

This was an observational, prospective, monocentric, physiological, cohort study, conducted from April to July 2020 in the high dependency respiratory care unit (HDRU) of Luigi Sacco University Hospital in Milan (Italy).

The study protocol (ClinicalTrials. gov: NCT04307459) followed the amended Declaration of Helsinki (2013), was approved by the local ethical committee (Comitato Etico Milano Area I; 17263/2020), and all recruited patients gave written informed consent.

Adult hospitalized patients with a virologically confirmed diagnosis of SARS-CoV-2 infection $[20,21]$ were considered eligible for the study and consecutively enrolled from April to May 2020 (for details on COVID-19 diagnosis, please see Supplementary Materials). Inclusion criteria were: 1) age $\geq 18$ years old; and 2) confirmed diagnosis of SARS-CoV-2 pneumonia. Patients were excluded from the study if unable to provide the informed consent or to perform repeatable lung function tests.

Anthropometrical, clinical characteristics, gas exchange parameters, and biochemistry were collected at admission. As soon as clinical stability was reached, while still hospitalized in the HDRU, patients underwent chest CT, blood gas analysis, spirometry, and DLco in the same day (Figure 1). The same routine was executed at the follow up visit, scheduled at 6 weeks ( \pm 5 days) post-discharge (Figure 1 ). In that instance, bodyplethysmography was performed instead of spirometry. At follow up, all patients had a negative SARS-CoV-2 nasopharyngeal swab, and lung function maneuvers were performed in the respiratory outpatient clinic as suggested by national and international safety recommendations $[22,23]$. 


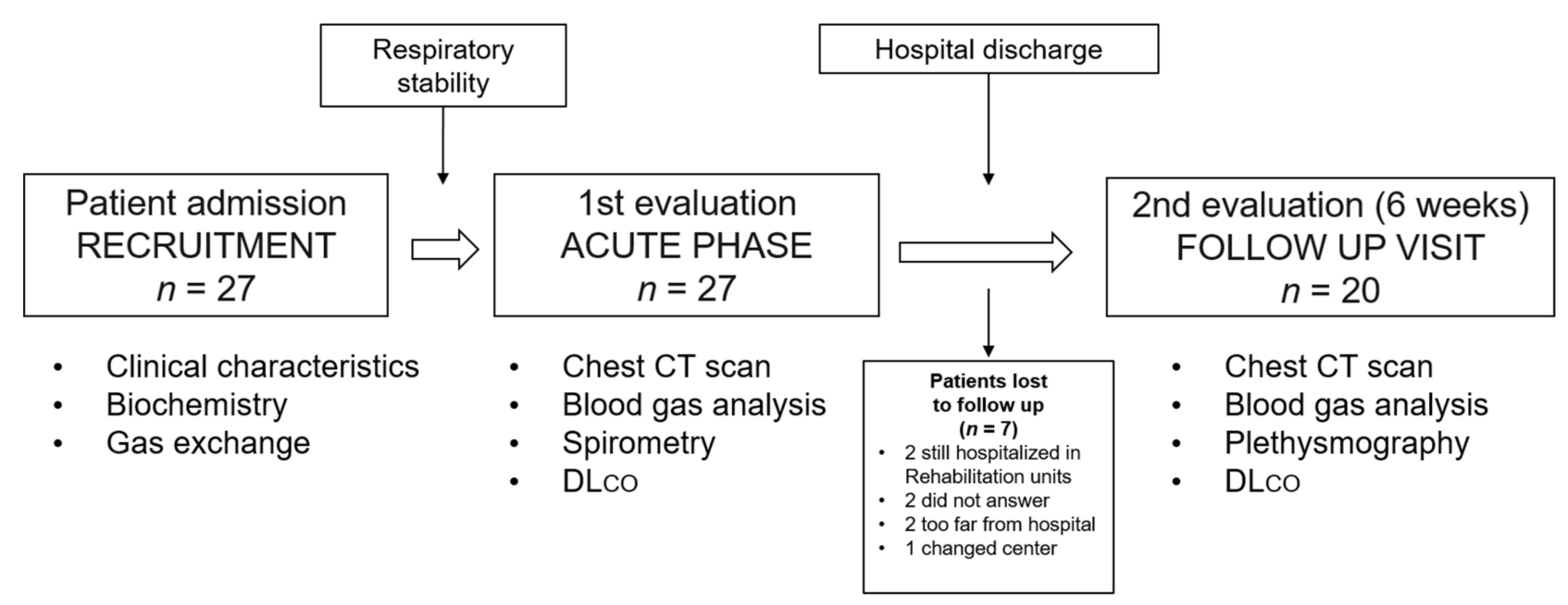

Figure 1. Flow chart of the study protocol. Seven patients were lost to follow up. DLco: lung diffusion capacity for carbon monoxide.

\subsection{Outcomes}

The primary objective was to investigate changes in lung function parameters (static, dynamic volumes, and DLCo), gas exchange, and chest CT findings assessed during hospitalization and after 6 weeks from hospital discharge.

Secondary objectives were: (1) to assess the relationship between gas exchange, lung function abnormalities and radiological patterns during hospitalization and during the recovery phase; and (2) to explore the determinants of DLco impairment during follow up.

\subsection{Procedures}

Gas exchange parameters were obtained from an arterial blood sample at rest. Details on gas exchange parameters and criteria used to grade respiratory failure are reported in the Supplementary Materials. During hospitalization, patients underwent lung function testing with spirometry and $\mathrm{DL}_{\mathrm{CO}}$ as soon as clinical stability was achieved. Clinical stability was considered when patients were able to tolerate a disconnection from oxygen therapy for the duration of the test and met the following criteria: $\mathrm{SpO}_{2} \geq 94 \%$ on $\leq 50 \% \mathrm{FiO}_{2}$; no need for continuous invasive or non-invasive respiratory support; absence of respiratory distress at rest; arterial systolic pressure $\geq 100 \mathrm{mmHg}$ and diastolic pressure $\geq 70 \mathrm{mmHg}$. An obstructive pattern was considered a forced expiratory volume in one second $\left(\mathrm{FEV}_{1}\right) /$ vital capacity (VC) ratio < Lower Limits of Normal (LLN) [24], and a restrictive pattern was a total lung capacity (TLC) $<80 \%$ predicted [24]. As plethysmography was available only during follow up, a pattern suggestive for restriction was defined as a $\mathrm{FEV}_{1} / \mathrm{VC} \geq 100 \%$ predicted associated with a $\mathrm{FEV}_{1}$ and $\mathrm{VC}<80 \%$ predicted. Lung function assessments were performed according to current American Thoracic Society/European Respiratory Society (ATS/ERS) recommendations [24,25] and are detailed in Supplementary Materials.

Multidetector chest $\mathrm{CT}$ was performed using either a 64-MDCT Lightspeed scanner (GE Medical Systems, Milwaukee, WI, USA) or a 64-MDCT Brilliance scanner (Philips Medical System, Amsterdam, Netherlands). Two experienced radiologists (N.F. and S.I.) retrospectively and independently reviewed the images on a PACS workstation (IMPAX, Agfa Healthcare, Mortsel, Belgium). Chest CT images were assessed for the presence of peripheral and multifocal ground glass opacities, consolidations, fibrosis, and crazy-paving patterns. The severity of disease was evaluated using the Total Severity Score (TSS), as previously reported [26,27]. CT scanning protocols and scoring method is reported in the Supplementary Materials.

Respiratory failure and pharmacological therapy were managed according to standard operating procedures and available recommendations [22,23,28-32] (Supplementary Materials). 


\subsection{Statistics}

The sample size was determined by the dynamics of the COVID-19 pandemic, and recruitment was stopped when the COVID-19 HDRU was closed in May 2020. At the time of the study, no evidence on changes in lung function parameters in patients with COVID19 pneumonia was available, so it was not possible to power the study. Qualitative variables were presented as absolute and relative (percentage) frequencies. The Shapiro Wilk test was used to assess normality of data distribution. Parametric and non-parametric quantitative variables were described with means (standard deviation, $\mathrm{SD}$ ) and medians (inter-quartile ranges, IQRs), respectively. McNemar's test was used to compare qualitative variables, while Student's t-test, Wilcoxon or Mann-Whitney U test were used to compare quantitative variables with normal or non-normal distribution, respectively. Relationships between variables were assessed by means of linear regression analysis. Predicted normal values of spirometric, plethysmographic, and DLco parameters were derived from Quanjer [33]. Clinical, functional, and radiological characteristics of patients with preserved ( $\geq 80 \%$ predicted) and impaired ( $<80 \%$ predicted) DLco were compared to assess predictors for DLco during follow up. Significant variables at the univariate analysis were used for the multiple regression analysis. The $\mathrm{D}$-dimer value was $\log 10$ transformed to fit a normal distribution. A two-tailed $p$-value $<0.05$ was considered statistically significant. All statistical computations were performed with "IBM SPSS Statistics for Windows", version 23 (IBM Corp, Armonk, NY, USA).

\section{Results}

A total of 27 patients were enrolled in the study, and 7 were lost at the follow up visit. Details about the reasons for drop-out are reported in the Supplementary Materials. Twenty patients (mean age 58.2, 70\% males) were included in the final analysis. Anthropometrical and clinical characteristics are reported in Table 1 . The most frequent comorbidity was arterial hypertension (55\%), 70\% of patients were lymphopenic, and the median (IQR) D-dimer at admission was 768 (374-3966) $\mathrm{mg} / \mathrm{L}$ fibrinogen equivalent units (FEU). Nine patients $(45 \%)$ had a D-dimer $>1000 \mathrm{mg} / \mathrm{L}$ FEU. The mean (SD) $\mathrm{PaO}_{2} / \mathrm{FiO}_{2}$ ratio at admission was $264(128) \mathrm{mmHg}$. A-a gradient was increased in all patients, and $95 \%$ had an A-a gradient $>100 \mathrm{mmHg}$. Continuous Positive Airway Pressure (CPAP) was employed in 13 patients (65\%); four of them eventually required invasive mechanical ventilation and were subsequently re-admitted in the HDRU once weaned from invasive mechanical ventilation (IMV). The first lung function assessment was performed on average 28.2 (13.5) days from symptoms onset and 9.1 (6.3) from admission (Table 1), while the follow up assessment was performed 43.8 (5.7) days from hospital discharge. Four patients were discharged on oxygen therapy and $12(60 \%)$ continued low molecular weight heparin (LMWH) after discharge.

Table 1. Anthropometric, clinical characteristics, in-hospital treatments, and outcomes of patients included in the final analysis.

\begin{tabular}{cc}
\hline & $\begin{array}{c}\text { Covid-19 Patients } \\
(\boldsymbol{n}=\mathbf{2 0})\end{array}$ \\
\hline Age at admission, years & $58.2(15.5)$ \\
\hline Males, $n(\%)$ & $14(70)$ \\
\hline BMI, $\mathrm{kg} / \mathrm{m}^{2}$ & $27.6(5.7)$ \\
\hline Ex and active smokers, $n(\%)$ & $4(20 \%)$ \\
\hline Arterial hypertension, $n(\%)$ & $11(55)$ \\
\hline Diabetes mellitus, $n(\%)$ & $2(10)$ \\
\hline Ischaemic heart disease, $n(\%)$ & $2(10)$ \\
\hline
\end{tabular}


Table 1. Cont.

\begin{tabular}{|c|c|c|}
\hline & & $\begin{array}{c}\text { Covid-19 Patients } \\
(n=20)\end{array}$ \\
\hline \multicolumn{3}{|c|}{ Chronic treatments } \\
\hline Angioten & lockers, $n(\%)$ & $5(20)$ \\
\hline & $n(\%)$ & $2(10)$ \\
\hline \multicolumn{3}{|c|}{ Blood count \& Biochemistry at admission } \\
\hline Lymphoc) & $10^{9} / \mu \mathrm{L}, n(\%)$ & $14(70)$ \\
\hline & FEU & $768(374-3966)$ \\
\hline D-dimer & L FEU, $n(\%)$ & $9(45)$ \\
\hline \multicolumn{3}{|c|}{ Gas exchange at admission } \\
\hline & & $7.45(0.03)$ \\
\hline & $\mathrm{Hg}$ & $42(35-49)$ \\
\hline & $\mathrm{mHg}$ & $264(128)$ \\
\hline \multirow{4}{*}{$\mathrm{PaO}_{2} / \mathrm{FiO}_{2}$} & $<100 \mathrm{mmHg}, n(\%)$ & $2(10)$ \\
\hline & 100-200 mmHg, $n(\%)$ & $6(30)$ \\
\hline & $201-300 \mathrm{mmHg}, n(\%)$ & $4(20)$ \\
\hline & $>300 \mathrm{mmHg}, n(\%)$ & $8(40)$ \\
\hline A-a & $\mathrm{mmHg}$ & $144(17-253)$ \\
\hline $\mathrm{A}-\mathrm{a} \mathrm{O}_{2} \mathrm{~g}$ & $\mathrm{mmHg}, n(\%)$ & $8(40)$ \\
\hline \multicolumn{3}{|c|}{ In-hospital treatments } \\
\hline Systemic & isolone, $n(\%)$ & $15(75)$ \\
\hline LMV & xis, $n(\%)$ & $11(55)$ \\
\hline LMW & lant, $n(\%)$ & $9(45)$ \\
\hline & & $13(65)$ \\
\hline & , days & $10.9(3.5)$ \\
\hline \multicolumn{3}{|c|}{ Outcomes } \\
\hline From sym & o 1st PFT, days & $28.2(13.5)$ \\
\hline From a & st PFT, days & $9.1(6.3)$ \\
\hline From d & d PFT, days & $43.8(5.7)$ \\
\hline Fror & FT, days & $52.4(6.4)$ \\
\hline Hosp & tion, days & $18.2(11.7)$ \\
\hline \multicolumn{3}{|c|}{ Treatments at discharge } \\
\hline & $(\%)$ & $4(20)$ \\
\hline & & $12(60)$ \\
\hline
\end{tabular}

Data are reported as means (standard deviation, SD), medians (inter-quartile range, IQR) and frequencies. BMI = body mass index; $\mathrm{LMWH}=$ low molecular weight heparin; $\mathrm{PFT}=$ pulmonary function test; $\mathrm{CPAP}=$ continuous positive airway pressure; $\mathrm{PEEP}=$ positive end expiratory pressure; $\mathrm{A}-\mathrm{a} \mathrm{O}_{2}$ gradient $=$ alveolar arterial oxygen gradient; $\mathrm{PaO}_{2} / \mathrm{FiO}_{2}=$ arterial partial oxygen pressure to fraction of inspired oxygen ratio; $\mathrm{PaCO}_{2}=$ arterial partial carbon dioxide pressure; $\mathrm{INR}$ = international normalized ratio; $\mathrm{FEU}$ = fibrinogen equivalent unit.

\subsection{Gas exchange and Lung Function}

At the time of first evaluation, the mean $\mathrm{PaO}_{2} / \mathrm{FiO}_{2}$ and median $\mathrm{A}-\mathrm{a} \mathrm{O}_{2}$ gradient were 316 (87) and 34 (19-101) $\mathrm{mmHg}$, respectively. At the follow up visit, none of the patients had respiratory failure, and the $\mathrm{A}-\mathrm{a} \mathrm{O}_{2}$ gradient was normalized in all patients (Table 2). 
Table 2. Gas exchange and lung function during hospitalization and at the follow up visit.

\begin{tabular}{|c|c|c|c|c|}
\hline & First PFT & Follow up PFT & Mean Difference (95\% CI) & $p$-Value \\
\hline \multicolumn{5}{|l|}{ Gas exchange } \\
\hline $\mathrm{FiO}_{2}, \%$ & $21(21-32)$ & $21(21-21)$ & - & 0.008 \\
\hline $\mathrm{pH}$ & $7.45(0.02)$ & $7.44(0.03)$ & $-0.01(-0.02-0.01)$ & 0.436 \\
\hline $\mathrm{PaCO}_{2}, \mathrm{mmHg}$ & $40(32-43)$ & $38(34-42)$ & - & 0.130 \\
\hline $\mathrm{PaO}_{2}, \mathrm{mmHg}$ & $78(71-88)$ & $90(87-100)$ & - & 0.002 \\
\hline HCO3-, mEq/L & $27.9(3.1)$ & $26.5(2.7)$ & $-1.4(-3.0-0.2)$ & 0.084 \\
\hline $\mathrm{PaO}_{2} / \mathrm{FiO}_{2}, \mathrm{mmHg}$ & $316(87)$ & $443(69)$ & $126(86-166)$ & $<0.001$ \\
\hline $\mathrm{A}-\mathrm{a} \mathrm{O}_{2}$ gradient, $\mathrm{mmHg}$ & $34(19-101)$ & $9(4-19)$ & - & $<0.001$ \\
\hline \multicolumn{5}{|l|}{ Spirometry } \\
\hline FVC, liters & $2.61(1.01)$ & $3.22(1.07)$ & $0.61(0.34-0.88)$ & $<0.001$ \\
\hline FVC, $\%$ predicted & $69.6(15.9)$ & $87.4(16.0)$ & $17.8(10.0-25.6)$ & $<0.001$ \\
\hline VC, liters & $2.73(1.09)$ & $3.35(1.10)$ & $0.62(0.39-0.86)$ & $<0.001$ \\
\hline VC, \% predicted & $71.7(16.9)$ & $87.4(16.2)$ & $15.7(9.9-21.5)$ & $<0.001$ \\
\hline $\mathrm{FEV}_{1}$, liters & $2.22(0.91)$ & $2.69(0.87)$ & $0.46(0.28-0.65)$ & $<0.001$ \\
\hline $\mathrm{FEV}_{1}, \%$ predicted & $75.1(18.9)$ & $91.3(15.2)$ & $16.3(8.9-23.6)$ & $<0.001$ \\
\hline $\mathrm{FEV}_{1} / \mathrm{VC}, \%$ predicted & $104.5(98.2-114.0)$ & $104.5(98.2-111.0)$ & - & 0.751 \\
\hline $\mathrm{FEV}_{1} / \mathrm{VC}<\mathrm{LLN}, n(\%)$ & $3(15)$ & $0(0)$ & - & 0.250 \\
\hline \multicolumn{5}{|l|}{ Body-plethysmography* } \\
\hline RV, liters & - & $1.74(0.50)$ & - & - \\
\hline $\mathrm{RV}, \%$ predicted & - & $82.5(28.8)$ & - & - \\
\hline IC, liters & - & $2.32(0.71)$ & - & - \\
\hline IC, \% predicted & - & $85.0(23.5)$ & - & - \\
\hline TLC, liters & - & $5.19(1.27)$ & - & - \\
\hline TLC, $\%$ predicted & - & $84.6(15.6)$ & - & - \\
\hline TLC $<80 \%$ predicted, $n(\%)$ & - & $7(35)$ & - & - \\
\hline $\mathrm{sRaw}_{\text {tot }}, \mathrm{kPa} \cdot \mathrm{s}$ & - & $0.83(0.32)$ & - & - \\
\hline sRaw $_{\text {tot }}, \%$ predicted & - & $75.0(28.0)$ & - & - \\
\hline \multicolumn{5}{|l|}{ Lung diffusion capacity } \\
\hline $\mathrm{DLco}, \mathrm{mmol} \cdot \mathrm{min}^{-1} \cdot \mathrm{kPa}^{-1}$ & $4.98(2.05)$ & $5.95(2.15)$ & $0.96(0.58-1.35)$ & $<0.001$ \\
\hline DLco, $\%$ predicted & $56.0(16.3)$ & $67.2(18.0)$ & $11.2(6.9-15.4)$ & $<0.001$ \\
\hline VA, liters & $3.88(1.34)$ & $4.54(1.38)$ & $0.66(0.39-0.92)$ & $<0.001$ \\
\hline VA, $\%$ predicted & $64.8(14.0)$ & $75.3(16.1)$ & $10.5(6.1-14.9)$ & $<0.001$ \\
\hline $\mathrm{VC} / \mathrm{VA}$ & $0.70(0.12)$ & $0.74(0.07)$ & $-0.04(-0.1-0.03)$ & 0.234 \\
\hline $\mathrm{KCO}, \mathrm{mmol} \cdot \mathrm{min}^{-1} \cdot \mathrm{kPa}^{-1} \cdot 1^{-1}$ & $1.32(0.36)$ & $1.32(0.28)$ & $-0.00(-0.09-0.92)$ & 0.981 \\
\hline $\mathrm{KCO}, \%$ predicted & $89.1(19.2)$ & $91.7(14.8)$ & $2.65(-3.2-8.5)$ & 0.358 \\
\hline
\end{tabular}

Data are reported as means (standard deviation, SD), medians (inter-quartile range, IQR) and frequencies. When appropriate, the

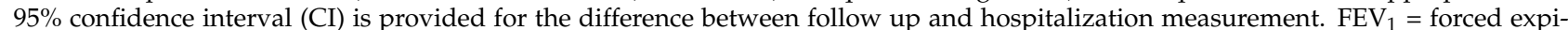
ratory volume in one second; FVC = forced vital capacity; VC = vital capacity; LLN = lower limits of normal; RV = residual volume; IC = inspiratory capacity; TLC = total lung capacity; sRAWtot = specific total airway resistances; DLco = lung diffusion capacity for carbon monoxide; $\mathrm{VA}=$ alveolar volume; $\mathrm{KCO}=$ transfer factor for carbon monoxide. *Plethysmographic parameters were measured only at the follow up visit. Two patients were unable to perform the test (one patient had hearing loss, one patient was claustrophobic). The data reported for plethysmography are referred to 18 patients. 
During hospitalization, $\mathrm{VC}$ and $\mathrm{FEV}_{1}$ were equally reduced (Table 2), which justified a median $\mathrm{FEV}_{1} / \mathrm{VC}$ ratio of 104.5 (98.2-114.0) \% predicted. Accordingly, 9 (45\%) patients had a spirometry suggestive for a restrictive pattern (Figure 2). DLco was reduced in $18(90 \%)$ patients, and moderately/severely reduced in 10 patients (50\%), mostly secondary to a reduction in VA ( $90 \%$ of patients) (Figure 2). At the follow up visit, all dynamic volumes improved (Table 2) and the $\mathrm{FEV}_{1} / \mathrm{VC}$ ratio remained unchanged, with 7 patients with persistent restrictive pattern. DLco improved from 56 (16.3) \% predicted to 67.2 (18.0) \% predicted $(p<0.001)$, but the proportion of patients with normal DLco and VA increased from 2 to $7(p=0.063)$ and from 2 to $9(p=0.016)$, respectively (Table 2 and Figure 2$)$.

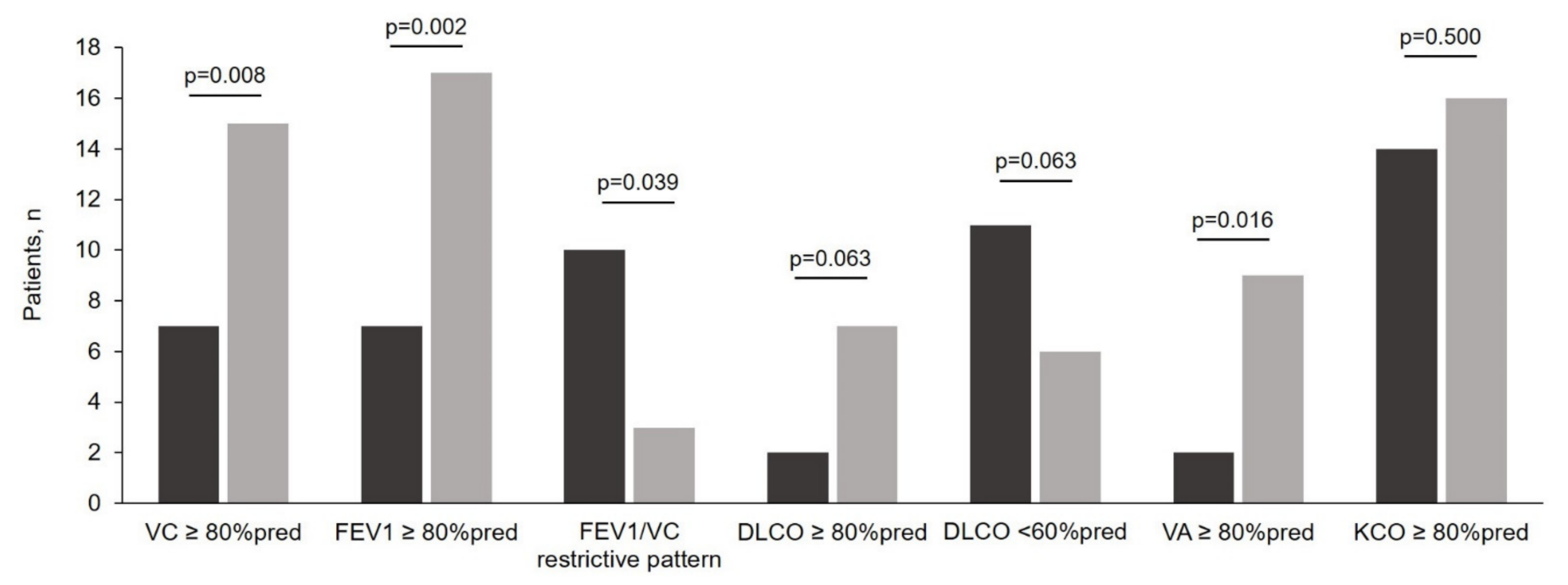

- Hospitalization $\quad$ Eollow up

Figure 2. Change in the proportion of patients with lung function alterations during hospitalization (dark grey columns) and at the follow up visit (light gray columns-6 weeks post-discharge). Restrictive pattern was considered the combination of a $\mathrm{FEV}_{1} / \mathrm{VC} \geq 100 \%$ predicted and a concomitant $\mathrm{FEV}_{1}$ and $\mathrm{VC}<80 \%$ predicted. DLco: lung diffusion capacity for carbon monoxide; $\mathrm{FEV}_{1}=$ forced expiratory volume in one second; $\mathrm{KCO}=$ transfer factor for carbon monoxide; $\mathrm{VA}=$ alveolar volume; $\mathrm{VC}=$ vital capacity.

\subsection{Chest CT}

The chest CT performed during hospitalization showed peripheral ground glass (pGGO), consolidation and fibrosis in $70 \%, 65 \%$, and $60 \%$ of patients, respectively. The mean TSS was 7.9 (4.0) (Supplementary Materials, Table S1). At 6 weeks post-discharge, consolidations were still present in only 2 patients $(10 \%)$, while the proportion of CT scans showing multifocal ground glass (mGGO) increased from $30 \%$ to $80 \%(p=0.002)$ (Figure 3 ). At follow up, TSS was numerically reduced (mean difference $(95 \% \mathrm{CI}): 1.6(-3.7-0.46)$; $p=0.118)$.

\subsection{Relationship between Lung Function, Radiological Patterns and Gas Exchange}

The presence of crazy paving and consolidations during the hospitalization was negatively correlated with ventilation inhomogeneity $(p=0.021)$, while consolidations were negatively correlated with DLco $(p=0.032)$ and VA $(p=0.011)$ (Supplementary Materials, Table S2). At the follow up CT, the persistence of consolidations negatively correlated with dynamic volumes and lung diffusion parameters (Supplementary Materials, Table S2). Severity of respiratory failure was positively correlated with DLco, VA, and VC (Figure 4). D-dimer values at admission were higher in patients with consolidations and were not associated with other chest CT abnormalities (Figure 5). 


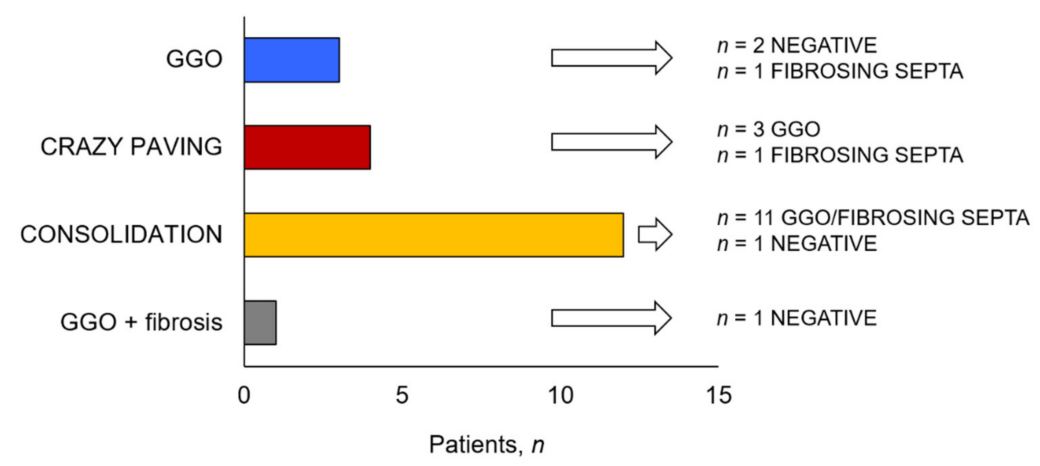

Figure 3. Changes in radiological patterns after 6 weeks from hospital discharge. GGO = ground glass opacities.
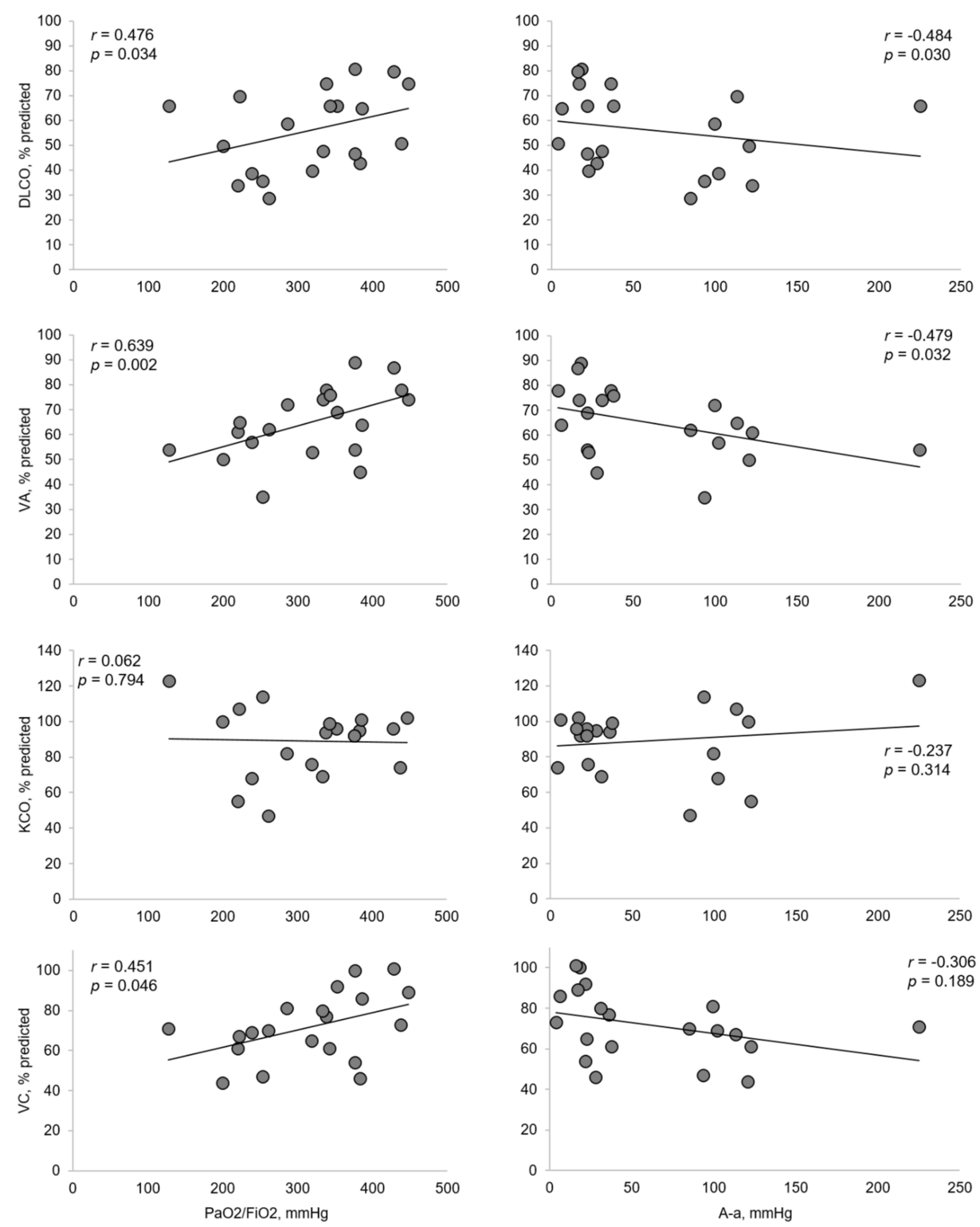

Figure 4. Relationship between $\mathrm{PaO}_{2} / \mathrm{FiO}_{2}$ ratio, $\mathrm{A}-\mathrm{a} \mathrm{O}_{2}$ gradient and lung diffusion parameters and VC. Correlations with FVC and $\mathrm{FEV}_{1}$ are not shown due to the strong association with VC ( $\mathrm{r}=0.859$ and $\mathrm{r}=0.829$, respectively) and between FVC and $\mathrm{FEV}_{1}(\mathrm{r}=0.884)$. DLco: lung diffusion capacity for carbon monoxide; $\mathrm{VA}=$ alveolar volume; $\mathrm{KCO}=$ transfer factor for carbon monoxide; $\mathrm{VC}=$ vital capacity. 


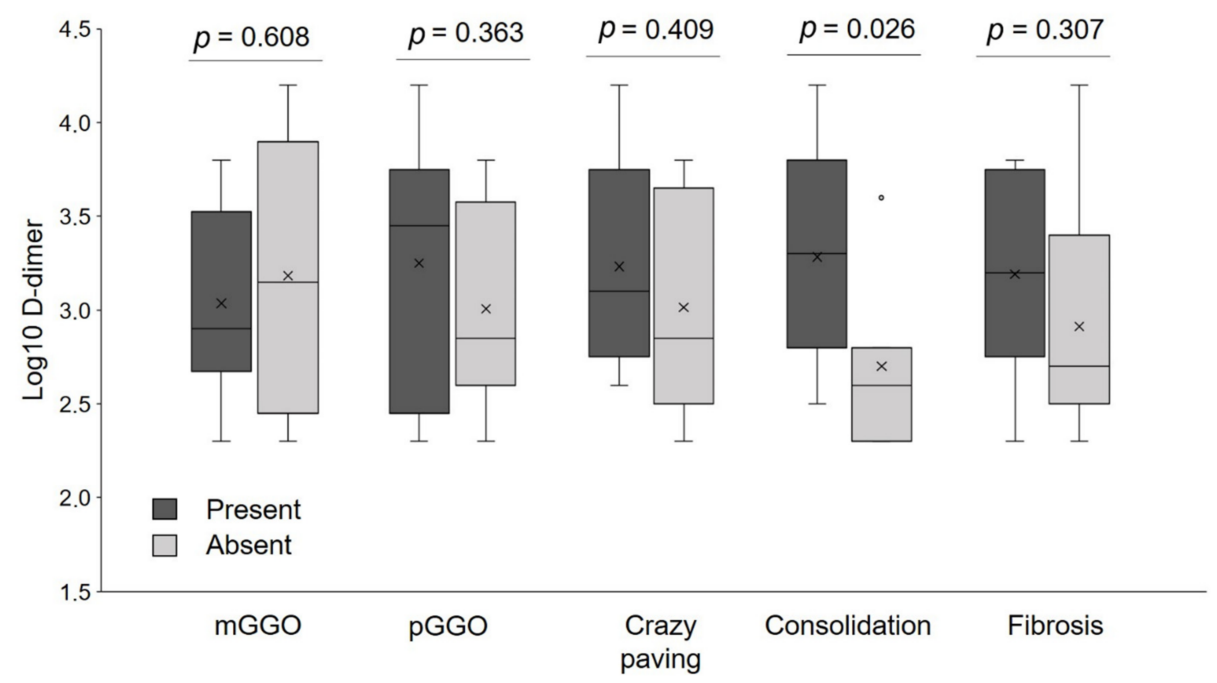

Figure 5. Distribution of D-dimer at admission in patients with different radiological pattern at the chest computed tomography (CT) performed at the time of first evaluation. Note that the only significant difference in D-dimer was in patients with and without parenchymal consolidations. Vertical lines represent the 75th and 25th percentile. Horizontal bars are medians. $X$ signs indicate means. pGGO = peripheral ground glass opacities; $\mathrm{mGGO}=$ multifocal ground glass opacities.

\subsection{Determinants of DLco during Follow up}

When compared with patients with preserved DLco at the follow up visit, patients with DLco $<80 \%$ predicted had higher D-dimer levels at admission $(p=0.008)$, and higher TSS ( $p=0.044)$ (Tables 3 and 4 ). D-dimer was significantly correlated with DLco and VA both during hospitalization and at follow up (Figure 6). A multiple regression analysis was performed to predict DLco at follow up from $\mathrm{FEV}_{1}, \mathrm{VC}$, TSS during hospitalization and D-dimer value at admission. The only variable that independently predicted DLco was D-dimer $(p=0.001)$, both when D-dimer was expressed as a continuous ( $\log _{10} \mathrm{D}$-dimer) or a dichotomous variable (D-dimer $>1000 \mathrm{mg} / \mathrm{L}$ FEU) (Table 5). This result did not change when hospital or home treatment with LMWH was added to the model (Supplementary Materials, Table S3), or the highest D-dimer value reached during hospitalization was considered. Figures 7 and 8 show an example of radiological and lung function evolution of two patients with impaired and normal DLco, respectively, during follow up.

Table 3. Clinical characteristics during hospitalization in patients with DLco $\geq 80 \%$ predicted and DLco $<80 \%$ predicted.

\begin{tabular}{|c|c|c|c|}
\hline & $\begin{array}{c}\text { DLco }<80 \% \text { Predicted } \\
\qquad(n=13)\end{array}$ & $\begin{array}{c}\text { DLco } \geq 80 \% \text { Predicted } \\
(n=7)\end{array}$ & $p$-Value \\
\hline Age, years & $58.5(14.6)$ & $57.7(18.3)$ & 0.921 \\
\hline BMI, $\mathrm{Kg} / \mathrm{m}^{2}$ & $24.9(23.3-27.2)$ & $31.1(22.7-33.0)$ & 0.311 \\
\hline Males, $n(\%)$ & $9(69)$ & $5(71)$ & 0.664 \\
\hline Arterial hypertension, $n(\%)$ & $7(54)$ & $4(57)$ & 0.630 \\
\hline Diabetes mellitus, $n(\%)$ & $2(15)$ & $0(0)$ & 0.411 \\
\hline Ischemic heart disease, $n(\%)$ & $1(8)$ & $1(14)$ & 0.589 \\
\hline \multicolumn{4}{|c|}{ Biochemistry at admission } \\
\hline White blood cells $\times 10^{9} / \mu \mathrm{L}$ & $9420(5010-14,515)$ & $5890(4560-7720)$ & 0.347 \\
\hline D-dimer, mg/L FEU & $3375(607-5699)$ & $394(200-733)$ & 0.008 \\
\hline D-dimer > 1000 mg/L FEU, $n(\%)$ & $9(69)$ & $0(0)$ & 0.004 \\
\hline
\end{tabular}


Table 3. Cont.

\begin{tabular}{|c|c|c|c|c|}
\hline & & $\begin{array}{c}\text { DLco }<80 \% \text { Predicted } \\
\qquad(n=13)\end{array}$ & $\begin{array}{c}\text { DLco } \geq 80 \% \text { Predicted } \\
(n=7)\end{array}$ & $p$-Value \\
\hline \multicolumn{2}{|c|}{ INR } & $1.25(1.13-1.42)$ & $1.17(1.10-1.27)$ & 0.516 \\
\hline \multicolumn{2}{|c|}{ Lymphocytes $\times 10^{9} / \mu \mathrm{L}$} & $1194(590)$ & $1311(726)$ & 0.701 \\
\hline \multicolumn{2}{|c|}{ Lymphocytes, \% } & $15.3(9.9)$ & $24.3(15.3)$ & 0.125 \\
\hline \multicolumn{2}{|c|}{$\mathrm{LDH}, \mathrm{U} / \mathrm{L}$} & $370(151)$ & $271(127)$ & 0.161 \\
\hline \multicolumn{5}{|c|}{ Interventions during hospitalization } \\
\hline \multicolumn{2}{|c|}{ CPAP, $n(\%)$} & $10(77)$ & $3(43)$ & 0.151 \\
\hline \multicolumn{2}{|c|}{ PEEP, mean (SD) } & $10.2(1.5)$ & $10.0(2.5)$ & 0.869 \\
\hline \multicolumn{2}{|c|}{ CPAP days, mean (SD) } & $11(4)$ & $11(3)$ & 0.957 \\
\hline \multicolumn{2}{|c|}{$\mathrm{LMWH}, n(\%)$} & $13(100)$ & $6(86)$ & 0.350 \\
\hline \multirow{2}{*}{ LMWH, $n(\%)$} & Treatment & $6(46)$ & $5(71)$ & 0.272 \\
\hline & Prophylaxis & $7(54)$ & $1(14)$ & 0.106 \\
\hline \multicolumn{2}{|c|}{ Corticosteroids, $n(\%)$} & $10(77)$ & $5(71)$ & 0.594 \\
\hline
\end{tabular}

Data are expressed as means (SD), median (IQR) and frequencies, as appropriate. Significant differences are in bold. BMI = body mass index; $\mathrm{LMWH}=$ low molecular weight heparin; $\mathrm{CPAP}=$ continuous positive airway pressure; $\mathrm{PEEP}=$ positive end expiratory pressure; $\mathrm{INR}=$ international normalized ratio; FEU = fibrinogen equivalent unit.

Table 4. Clinical and functional characteristics during hospitalization and at follow up in patients with DLCO $\geq 80 \%$ predicted and DLCO $<80 \%$ predicted.

\begin{tabular}{|c|c|c|c|c|c|c|}
\hline & \multicolumn{2}{|c|}{$\mathrm{DL}_{\mathrm{CO}} \geq 80 \%$ Predicted $(n=13)$} & \multicolumn{2}{|c|}{$\mathrm{DL}_{\mathrm{CO}}<80 \%$ Predicted $(n=7)$} & \multirow[b]{2}{*}{$p$-Value } & \multirow[b]{2}{*}{$p$-Value * } \\
\hline & $\begin{array}{c}\text { During } \\
\text { Hospitalisation }\end{array}$ & Follow up * & $\begin{array}{c}\text { During } \\
\text { Hospitalisation }\end{array}$ & Follow up * & & \\
\hline \multicolumn{7}{|c|}{ Chest CT patterns } \\
\hline TSS, points & $10.2(6.0)$ & $6.4(4.0)$ & $7.4(3.5)$ & $6.0(3.3)$ & 0.044 & 0.797 \\
\hline Peripheral GGO, n (\%) & $9(69)$ & $5(38)$ & $5(71)$ & $4(57)$ & 0.664 & 0.370 \\
\hline Multifocal GGO, n (\%) & $5(38)$ & $10(77)$ & $1(14)$ & $6(86)$ & 0.277 & 0.561 \\
\hline Crazy paving, n (\%) & $4(31)$ & $1(8)$ & $2(29)$ & $0(0)$ & 0.664 & 0.650 \\
\hline Consolidation, $\mathrm{n}(\%)$ & $9(69)$ & $2(15)$ & $4(57)$ & $0(0)$ & 0.474 & 0.411 \\
\hline Fibrosis, n (\%) & $8(61)$ & $10(77)$ & $4(57)$ & $4(57)$ & 0.608 & 0.336 \\
\hline Post vs. ant. Prev., n (\%) & $10(77)$ & $10(77)$ & $6(86)$ & $6(86)$ & 0.561 & 0.561 \\
\hline \multicolumn{7}{|c|}{ Gas exchange parameters } \\
\hline $\mathrm{FiO}_{2}, \%$ & $24(21-32)$ & $21(0)$ & $21(21-35)$ & $21(0)$ & 0.316 & $n / \mathrm{a}$ \\
\hline $\mathrm{pH}$ & $7.47(7.44-7.48)$ & $\begin{array}{c}7.45 \\
(7.42-7.47) \\
\end{array}$ & $7.43(7.42-7.44)$ & $\begin{array}{c}7.44 \\
(7.41-7.45) \\
\end{array}$ & 0.853 & 0.211 \\
\hline $\mathrm{PaCO}_{2}, \mathrm{mmHg}$ & $39.8(4.6)$ & $37.8(4.1)$ & $42.1(8.0)$ & $39.4(5.5)$ & 0.404 & 0.457 \\
\hline $\mathrm{PaO}_{2}, \mathrm{mmHg}$ & $76.5(8.3)$ & $89.8(13.1)$ & $83.1(12.4)$ & $99.1(15.9)$ & 0.169 & 0.173 \\
\hline $\mathrm{HCO}^{-}, \mathrm{mmol} / \mathrm{L}$ & $28.3(2.6)$ & $26.5(2.2)$ & $27.2(4.0)$ & $26.4(3.6)$ & 0.452 & 0.933 \\
\hline $\mathrm{PaO}_{2} / \mathrm{FiO}_{2}, \mathrm{mmHg}$ & $300(74)$ & $427(62)$ & $343(109)$ & $472(76)$ & 0.325 & 0.173 \\
\hline $\mathrm{A}-\mathrm{a} \mathrm{O}_{2}$ gradient, $\mathrm{mmHg}$ & $38(25-108)$ & $12(6-20)$ & $18(16-99)$ & $0.0(0-0)$ & 0.126 & 0.056 \\
\hline \multicolumn{7}{|c|}{ Lung function } \\
\hline FVC, \%predicted & $63.1(12.1)$ & $82.8(15.7)$ & $81.6(15.8)$ & $95.5(13.8)$ & 0.023 & 0.082 \\
\hline $\mathrm{FEV}_{1}, \%$ predicted & $67.6(15.1)$ & $86.5(15.8)$ & $88.6(18.1)$ & $100.4(9.2)$ & 0.012 & 0.047 \\
\hline
\end{tabular}


Table 4. Cont.

\begin{tabular}{|c|c|c|c|c|c|c|}
\hline & \multicolumn{2}{|c|}{$\mathrm{DL}_{\mathrm{CO}} \geq 80 \%$ Predicted $(n=13)$} & \multicolumn{2}{|c|}{$\mathrm{DL}_{\mathrm{CO}}<80 \%$ Predicted $(n=7)$} & \multirow[b]{2}{*}{$p$-Value } & \multirow[b]{2}{*}{$p$-Value * } \\
\hline & $\begin{array}{c}\text { During } \\
\text { Hospitalisation }\end{array}$ & Follow up * & $\begin{array}{c}\text { During } \\
\text { Hospitalisation }\end{array}$ & Follow up * & & \\
\hline VC, \%predicted & $62.6(11.8)$ & $82.8(17.3)$ & $88.6(10.6)$ & $96.1(9.8)$ & $<0.001$ & 0.077 \\
\hline $\mathrm{FEV}_{1} / \mathrm{VC}, \%$ predicted & $105.8(18.5)$ & $105.1(10.1)$ & $106.4(13.6)$ & $104.7(9.1)$ & 0.935 & 0.935 \\
\hline TLC, \%predicted & - & $80.0(15.5)$ & - & $91.9(13.7)$ & - & 0.119 \\
\hline TLC $>80 \%$ predicted, n $(\%)$ & & $6(54.5)$ & & $1(14.3)$ & & 0.112 \\
\hline IC, \%predicted & - & $78.0(23.1)$ & - & $96.0(21.2)$ & - & 0.116 \\
\hline RV, \%predicted & - & $74.8(24.5)$ & - & $94.6(32.6)$ & - & 0.162 \\
\hline DLco, \%predicted & $48.3(14.2)$ & $57.5(14.1)$ & $70.2(8.4)$ & $85.1(6.6)$ & 0.002 & $<0.001$ \\
\hline $\mathrm{KCO}, \%$ predicted & $83.5(20.5)$ & $86.4(14.7)$ & $98.6(12.5)$ & $101.8(9.1)$ & 0.096 & 0.022 \\
\hline VA, \%predicted & $60.6(13.4)$ & $69.4(16.1)$ & $72.7(12.3)$ & $86.4(8.9)$ & 0.064 & 0.020 \\
\hline VA/TLC & & $0.87(0.13)$ & & $0.92(0.1)$ & & 0.394 \\
\hline $\mathrm{VA} / \mathrm{VC}$ & $0.67(0.12)$ & $0.75(0.06)$ & $0.75(0.09)$ & $0.70(0.08)$ & 0.159 & 0.102 \\
\hline
\end{tabular}

Data are expressed as means (SD), median (IQR) and frequencies, as appropriate. Significant differences are in bold. TSS = total severity score; $\mathrm{GGO}=$ ground glass; $\mathrm{A}-\mathrm{a} \mathrm{O}_{2}$ gradient $=$ alveolar arterial oxygen gradient; $\mathrm{PaO}_{2} / \mathrm{FiO}_{2}=$ arterial partial oxygen pressure to fraction of inspired oxygen ratio; $\mathrm{PaCO}_{2}=$ arterial partial carbon dioxide pressure; $\mathrm{FEV}_{1}=$ forced expiratory volume in one second; $\mathrm{FVC}=$ forced vital capacity; VC = vital capacity; $\mathrm{LLN}=$ lower limits of normal; RV = residual volume; IC $=$ inspiratory capacity; TLC = total lung capacity; sRAWtot = specific total airway resistances; $\mathrm{DLco}=$ lung diffusion capacity for carbon monoxide; VA = alveolar volume; $\mathrm{KCO}=$ transfer factor for carbon monoxide. ${ }^{*}$ comparison between the follow up observations.
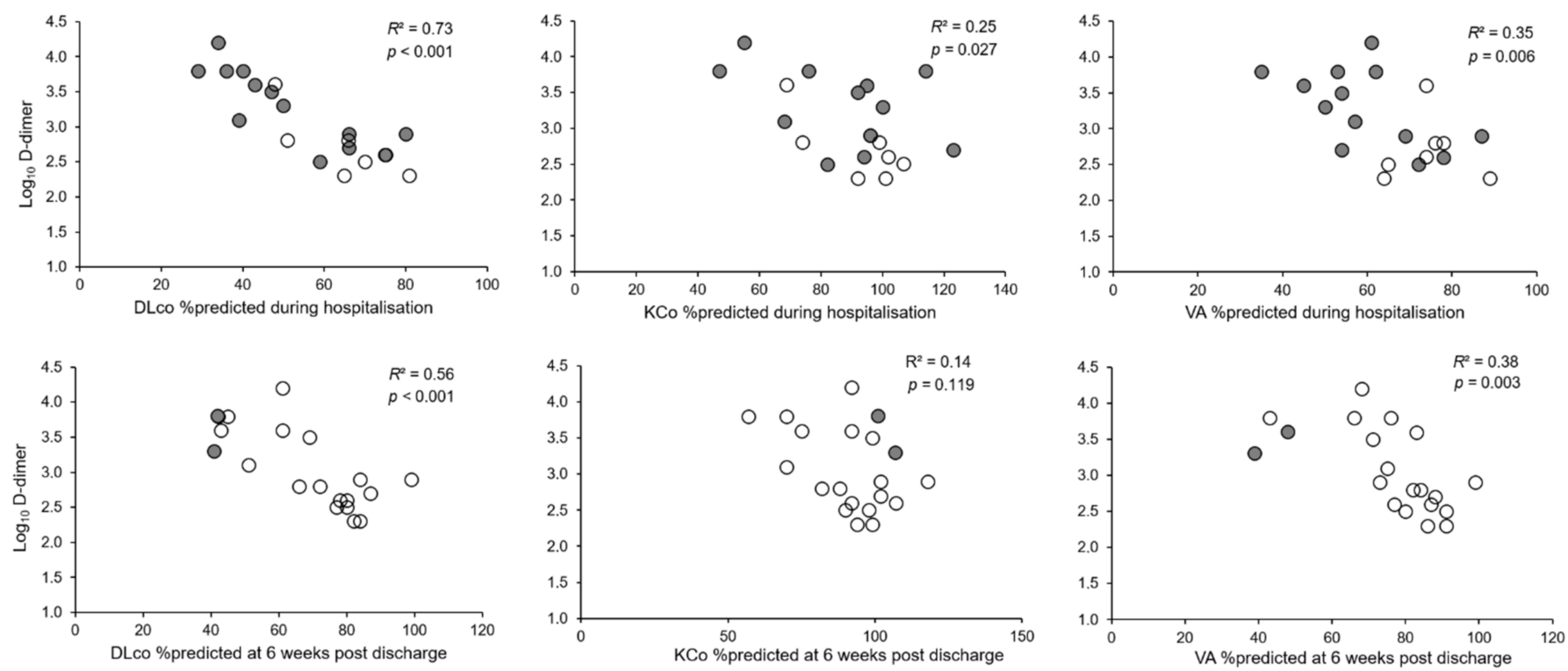

Figure 6. Relationship between D-dimer values at admission and DLco, KCO, and VA \% predicted at first assessment during hospitalisation (upper panels) and after 6 weeks from hospital discharge (lower panels). Full circles are patients with consolidations at the chest CT perfromed the same day of the DLco, and empty circles indicate patients without consolidations. DLCo = lung diffusion capacity for carbon monoxide; $\mathrm{KCO}=$ transfer factor for carbon monoxide; $\mathrm{VA}=$ alveolar volume. 
Table 5. Predicting factors for DLco in the post-discharge period.

\begin{tabular}{|c|c|c|c|c|}
\hline & Unstandardized Coefficient & Standardized Coefficient & $95 \%$ CI & $p$-Value \\
\hline \multicolumn{5}{|l|}{$\begin{array}{c}\text { Model } 1 \\
\left.\text { (adj } R^{2}: 0.786\right)\end{array}$} \\
\hline $\mathrm{FEV}_{1} \%$ pred & -0.043 & -0.050 & $-0.442-0.355$ & 0.820 \\
\hline VC \%pred & 0.227 & 0.235 & $-0.294-0.748$ & 0.368 \\
\hline TSS, points & -0.623 & -0.155 & $-1.877-0.632$ & 0.307 \\
\hline $\mathrm{Lg}_{10}$ D-dimer & -18.675 & -0.655 & $\begin{array}{c}-28.373- \\
-9.076\end{array}$ & 0.001 \\
\hline \multicolumn{5}{|l|}{$\begin{array}{c}\text { Model } 2 \\
\left.\text { (adj } R^{2}: 0.786\right)\end{array}$} \\
\hline $\mathrm{FEV}_{1} \%$ pred & -0.026 & -0.030 & $-0.426-0.373$ & 0.868 \\
\hline VC \%pred & 0.161 & 0.167 & $-0.374-0.697$ & 0.172 \\
\hline TSS, points & -0.426 & -0.106 & $-1.710-0.859$ & 0.209 \\
\hline D-dimer $\geq 1000 \mathrm{FEU}$ & -22.849 & -0.716 & $\begin{array}{l}-34.594- \\
-11.105\end{array}$ & 0.001 \\
\hline
\end{tabular}

Multiple regression analysis models for predicting DLco \% predicted at follow up. Having a D-dimer $>1000$ at admission is associated with a DLco reduction of $22 \%$ at 6 weeks post-discharge. Variables significantly different at the univariate analysis $\left(\mathrm{FEV}_{1}, \mathrm{VC}, \mathrm{TSS}\right.$, and D-dimer) were used for the linear regression analysis. FVC was excluded from the model because it is intrinsically highly correlated with VC (see legend of Figure 4 for details). CI = confidence interval; $\mathrm{FEV}_{1}=$ forced expiratory volume in one second; $\mathrm{VC}=$ vital capacity; TSS $=$ total severity score
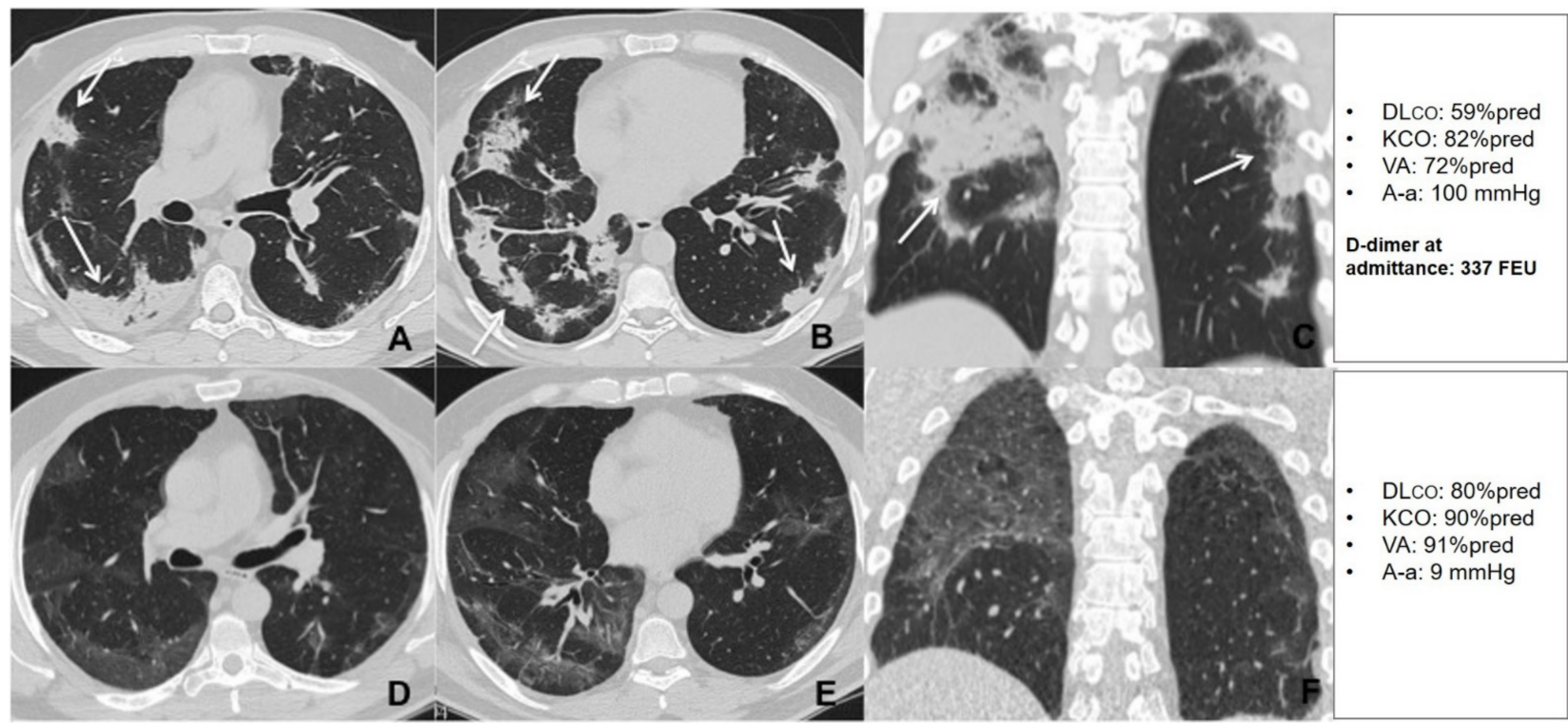

Figure 7. Unenhanced thin section axial (A,B) and coronal (C) CT images of the lungs obtained the 10th day post-admission in a 51-year-old man with no previous exposure to cigarette smoke, no cormorbidities, that was admitted to the high dependency respiratory care unit (HDRU) with severe respiratory failure $\left(\mathrm{PaO}_{2} / \mathrm{FiO}_{2} 182 \mathrm{mmHg}\right)$ and treated with continuous positive airway pressure (CPAP) for 10 days. The images show peripheral consolidations (arrows) in both lungs, with predilection for posterior areas. Unenhanced thin section axial (D,E) and coronal (F) CT images of the same patient 6 weeks post-discharge show bilateral peripheral GGO with resolution of previous consolidations. The patient had a low D-dimer at admission and showed a significant improvement in his DLco during the recovery period. 


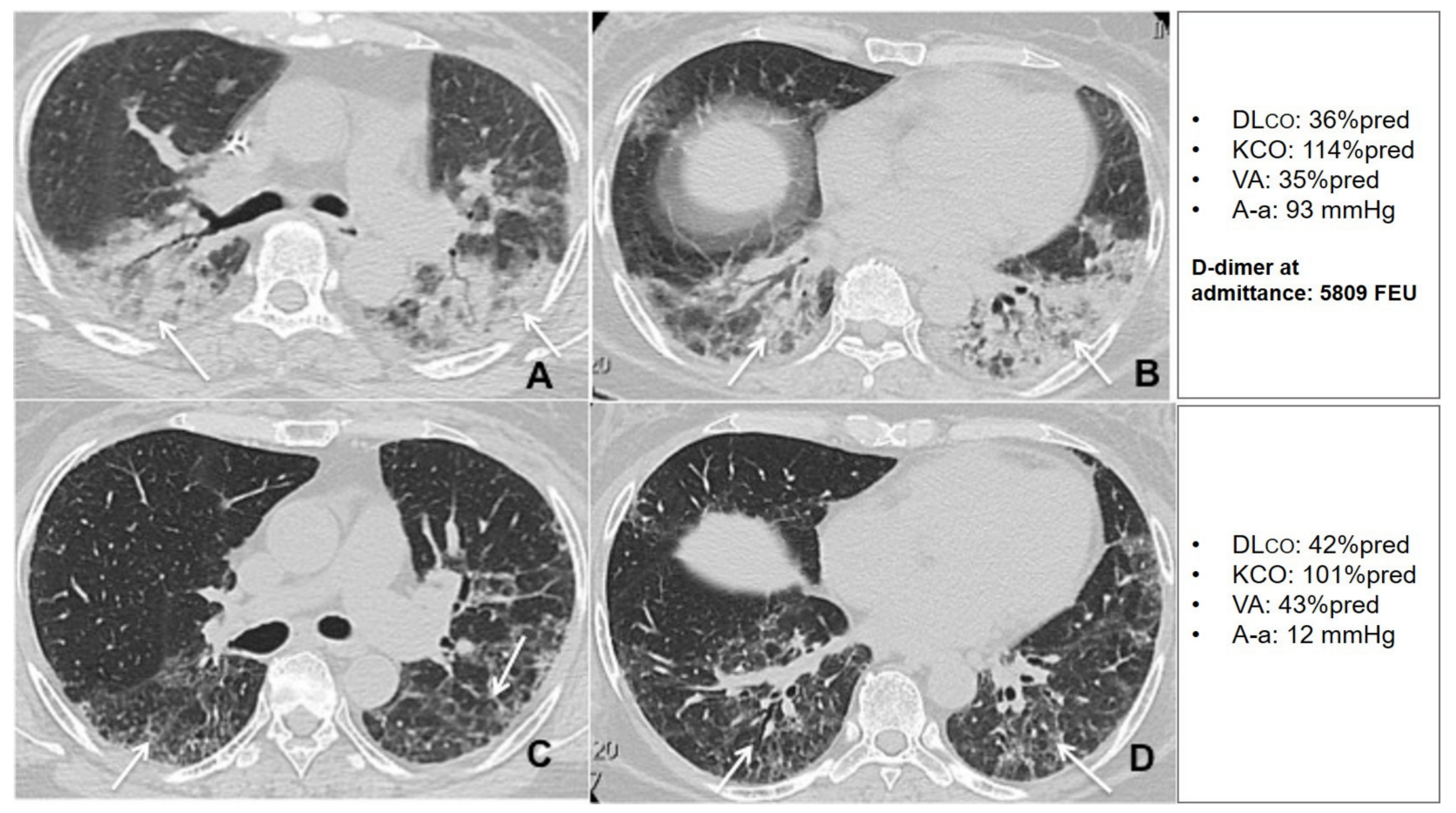

Figure 8. Unenhanced thin section axial (A,B) CT images of the lungs obtained the 12th day post-admission in a 57-year-old woman with arterial hypertension and no previous exposure to cigarette smoke, that was admitted to the HDRU with a $\mathrm{PaO}_{2} / \mathrm{FiO}_{2}$ of $210 \mathrm{mmHg}$, rapidly worsened her respiratory status and needed invasive mechanical support for 8 days. The images show peripheral posterior GGO and consolidations in both lungs, with predilection for posterior areas (arrows). Unenhanced axial (C,D) CT images of the same patient 6 weeks post-discharge show residual interlobular septal thickening with intralobular lines (arrows) with resolution of previous GGO and consolidation. The patient had a high D-dimer at admission and showed very limited changes in DLco during the recovery period.

\section{Discussion}

The main results of the present study can be summarized as follows: (1) during the acute phase patients with COVID-19 pneumonia showed a prevalent restrictive functional pattern, characterized by a concomitant reduction in $\mathrm{VC}$ and $\mathrm{FEV}_{1}$ and paralleled by an impairment in DLco; (2) the reduction in dynamic volumes and DLco was correlated with disease severity and was associated with the presence of lung consolidations both during the acute phase and after 6 weeks of follow up; (3) at 6 weeks post-discharge, DLco was at least mildly reduced in $65 \%$ of cases, while respiratory failure was completely resolved in all patients; and (4) D-dimer at admission was the only parameter that predicted the DLco value during the recovery phase.

Pulmonary sequelae in survivors from COVID-19 pneumonia have been a matter of intense discussion [16,34]. In fact, all studies, but one [12], performed a single lung functional assessment [9-11,13-16], limiting any hypothesis on the potential evolution and determinants of lung function impairment in these patients.

To our knowledge, this is the first study to show that, during the acute phase (about 9 days after hospital admission), about 50\% of patients with COVID-19 pneumonia experience a restrictive respiratory pattern, with an equal and parallel reduction of $\mathrm{FEV}_{1}$, VC, and FVC, while only $10 \%$ of patients had a preserved DLco. The latter finding was sustained by a positive correlation with parenchymal consolidations at the chest $\mathrm{CT}$ and, in line with previous reports [12,16], it was directly associated with the fall in $\mathrm{PaO}_{2} / \mathrm{FiO}_{2}$ ratio observed in many patients. Indeed, testing patients just before hospital discharge, Fumagalli and colleagues [12] found a frequent reduction in FVC and FEV 1 , while Mo and coworkers [16] observed a restrictive disease in $25 \%$ and a reduced DLco in $47.2 \%$ of patients, a proportion that was doubled in case of severe disease. 
After 6 weeks from hospital discharge, we observed a reduced DLco in $65 \%$ of patients, in line with the majority of available reports [10,13]. Contrary to previous studies [14], we did not find signs of airflow obstruction or small airways involvement in our cohort, probably due to the low prevalence of patients affected by chronic obstructive diseases and smoke exposure.

During follow up, ground glass opacities and septal fibrosis characterized $>70 \%$ of patients, while only three subjects had a chest CT without any abnormality at the follow up visit. These data are in partial agreement with available reports. In fact, Rogliani and coworkers found almost no radiological abnormalities after 4 weeks of follow up in patients with mild to moderate COVID-19 pneumonia [10]. On the other hand, Shah and colleagues detected residual GGO in $83 \%$ of patients with moderate to critical disease [14]. Indeed, in contrast with our cohort, only $11 \%$ of the patients enrolled by Rogliani et al. had a severe respiratory failure, thus expecting the possibility of milder lung involvement and faster radiological restitutio ad integrum [10].

Some authors speculated that, in patients recovering from COVID-19 pneumonia, the observed reduction in DLco could be secondary to the development of fibrosis [11]. Unexpectedly, we did not find an association between $\mathrm{DL}_{\mathrm{CO}}$ and lung fibrosis. Nevertheless, patients with a lower DLco at follow up had lower dynamic volumes, a higher prevalence of lung restriction ( $54 \%$ vs. $14 \%$ ), and a worse TSS and higher D-dimer values during hospitalization compared with patients with preserved DLco.

We found that D-dimer at admission had a strong association with DLco and VA, and independently predicted DLco values during follow up. It is well recognized that patients with COVID-19 pneumonia are at high risk of micro and macro thrombotic events $[3,35,36]$. In the latter case, an increase in D-dimer would be expected to be related with $\mathrm{KCO}$, an index of alveolar-capillary membrane dysfunction [37]. However, D-dimer showed only a weak correlation with $\mathrm{KCO}$, and no association was found with in-hospital or home treatment with LMWH. We, therefore, hypothesize that the dynamics of DLco reduction and its relationship with D-dimer could be one or a combination of the following. First, a $\mathrm{KCO}$ overestimation due to the assessment of DLco in patients with a functional restrictive pattern [38] may have masked the possible persistence of a damage to the vascular component. Second, the reduction of static volumes, and hence the persistence of VA impairment during the recovery phase, excluding respiratory muscles contractile abnormalities, could be secondary to the lung anatomical derangement, which has been shown to persist up to $4-5$ months after hospital discharge in patients with COVID-19 pneumonia [11]. Third, considering the low prevalence of patients with history of chronic lung diseases and obesity in our cohort, a re-distribution of the regional ventilation/perfusion ratio might have partially compensated the gas transfer efficiency, maintaining satisfactory KCO levels in some patients.

Despite the physiological and pivotal design of our study, we believe that these observations can provide useful hints from the clinical point of view. In fact, we suggest that during follow up, patients that survived a COVID-19 pneumonia should be monitored with lung function tests, particularly with DLco in case of higher D-dimer levels during hospitalization.

The present study has limitations. First, the small sample size, limited by the reduction of COVID-19 cases during May 2020 in our region. Second, the study enrolled patients with moderate-to-severe disease, limiting the generalizability of the study findings to milder forms of SARS_CoV-2 infection. Third, the lack of assessment of static volumes during the first evaluation might have caused an underestimation of the real number of restricted patients during the acute phase. Finally, the lack of lung function data in the period preceding the SARS-CoV-2 pandemic may have limited the estimation of the actual pathophysiological effects of COVID-19 pneumonia; indeed, the number of patients with such measurements would be very low, considered the very low prevalence of patients with chronic respiratory diseases in our sample. 


\section{Conclusions}

In conclusion, for the first time, lung volumes and DLco in patients hospitalized with moderate to severe COVID-19 pneumonia have been assessed both during hospitalization and follow up. Patients acutely experience a mild to severe reduction in DLco that can persist up to 6 weeks post-discharge. Moreover, DLco impairment during follow up is predicted by D-dimer values at hospital admission, confirming the role of D-dimer as a marker of severity of lung involvement. In COVID-19 patients, radiological and functional parameters appear to follow different dynamics, and, currently, the proportion of patients that would suffer for permanent functional abnormalities (e.g., fibrosis) and which could be the effect of reduced DLco on exercise capacity or daily life activities remains largely unknown. Long-term follow up studies will be required to address this question.

Supplementary Materials: The following are available online at https:/ /www.mdpi.com/2077-0 383/10/5/1021/s1, Table S1. Radiological patterns during the acute phase and after 6 weeks postdischarge, Table S2. Relationship between lung function parameters and chest CT patterns during the acute phase and at 6 weeks post-discharge, Table S3. Multiple regression analysis for predicting DLCO \%predicted at follow up, Table S4. STROBE 2007 (v4) Statement-Checklist of items that should be included in reports of cohort studies, Supplementary Materials and Methods.

Author Contributions: Conceptualization, P.S., M.R., and D.R.; Data curation, P.S, N.F., M.S.,S.P., E.F., A.A. P.G., S.I., M.R., and D.R.; Formal analysis, P.S., N.F., S.I., M.R., and D.R.; Investigation, M.S., S.P., E.F., A.A., P.G., M.R., and D.R.; Methodology, P.S., M.R., and D.R.; Software, N.F. and S.I. Supervision, P.S., M.R., and D.R.; Validation, N.F. and S.I.; Visualization, M.R.; Writing-original draft, P.S., P.G., and D.R.; Writing-review \& editing, P.S., N.F., M.S., S.P., E.F., A.A., S.I., and M.R. All authors have read and agreed to the published version of the manuscript.

Funding: This research received no external funding.

Institutional Review Board Statement: The study was conducted according to the guidelines of the Declaration of Helsinki, and approved by the local ethical committee (Comitato Etico Milano Area I; 17263/2020). All recruited patients gave written informed consent.

Informed Consent Statement: Informed consent was obtained from all subjects involved in the study.

Data Availability Statement: The anonymized datasets used and analyzed during the current study are available from the corresponding Author on reasonable request.

Conflicts of Interest: The authors declare no conflict of interest.

\section{References}

1. World Health Organization (WHO) Weekly Epidemiological Update. 2020. Available online: https://www.who.int/publications / $\mathrm{m} /$ item/weekly-epidemiological-update---3-november-2020 (accessed on 16 November 2020).

2. European Centre for Disease Prevention and Control. Data on Hospital and ICU Admission Rates and Current Occupancy for COVID-19. 2020. Available online: https:/ / www.ecdc.europa.eu/en/publications-data/download-data-hospital-and-icuadmission-rates-and-current-occupancy-covid-19 (accessed on 16 November 2020).

3. Carsana, L.; Sonzogni, A.; Nasr, A.; Rossi, R.S.; Pellegrinelli, A.; Zerbi, P.; Rech, R.; Colombo, R.; Antinori, S.; Corbellino, M.; et al. Pulmonary post-mortem findings in a series of COVID-19 cases from northern Italy: A two-centre descriptive study. Lancet Infect. Dis. 2020, 20, 1135-1140. [CrossRef]

4. Liu, X.; Zhou, H.; Zhou, Y.; Wu, X.; Zhao, Y.; Lu, Y.; Tan, W.; Yuan, M.; Ding, X.; Zou, J.; et al. Temporal radiographic changes in COVID-19 patients: Relationship to disease severity and viral clearance. Sci. Rep. 2020, 10, 10263. [CrossRef]

5. Yao, Y.; Cao, J.; Wang, Q.; Shi, Q.; Liu, K.; Luo, Z.; Chen, X.; Chen, S.; Yu, K.; Huang, Z.; et al. D-dimer as a biomarker for disease severity and mortality in COVID-19 patients: A case control study. J. Intensive Care 2020, 8, 49. [CrossRef]

6. Viecca, M.; Radovanovic, D.; Forleo, G.B.; Santus, P. Enhanced platelet inhibition treatment improves hypoxemia in patients with severe Covid-19 and hypercoagulability. A case control, proof of concept study. Pharmacol. Res. 2020, 158, 104950. [CrossRef]

7. Pan, F.; Zheng, C.; Ye, T.; Li, L.; Liu, D.; Li, L.; Hesket, R.L.; Yang, L. Different computed tomography patterns of Coronavirus Disease 2019 (COVID-19) between survivors and non-survivors. Sci. Rep. 2020, 10, 11336. [CrossRef]

8. Liu, C.; Ye, L.; Xia, R.; Zheng, X.; Yuan, C.; Wang, Z.; Lin, R.; Shi, D.; Gao, Y.; Yao, J.; et al. Chest computed tomography and clinical follow-up of discharged patients with COVID-19 in Wenzhou City, Zhejiang, China. Ann. Am. Thorac. Soc. 2020, 17, 1231-1237. [CrossRef] 
9. Rogliani, P.; Calzetta, L.; Coppola, A.; Puxeddu, E.; Sergiacomi, G.; D'Amato, D.; Orlacchio, A. Are there pulmonary sequelae in patients recovering from COVID-19? Respir. Res. 2020, 21, 286. [CrossRef]

10. Huang, Y.; Tan, C.; Wu, J.; Chen, M.; Wang, Z.; Luo, L.; Zhou, X.; Liu, X.; Huang, X.; Yuan, S.; et al. Impact of coronavirus disease 2019 on pulmonary function in early convalescence phase. Respir. Res. 2020, 21, 163. [CrossRef]

11. Zhao, Y.M.; Shang, Y.M.; Song, W.B.; Li, Q.Q.; Xie, H.; Xue, Q.; Jia, J.; Li, L.; Mao, H.; Zhou, X.; et al. Follow-up study of the pulmonary function and related physiological characteristics of COVID-19 survivors three months after recovery. EClinicalMedicine 2020, 25, 100463. [CrossRef]

12. Fumagalli, A.; Misuraca, C.; Bianchi, A.; Borsa, N.; Limonta, S.; Maggiolini, S.; Bonardi, D.R.; Corsonello, A.; Di Rosa, M.; Soraci, L.; et al. Pulmonary function in patients surviving to COVID-19 pneumonia. Infection 2020, 49, 1-5. [CrossRef] [PubMed]

13. Van der Sar-van der Brugge, S.; Talman, S.; Boonman-de Winter, L.; de Mol, M.; Hoefman, E.; van Etten, R.W.; De Backer, I.C. Pulmonary function and health-related quality of life after COVID-19 pneumonia. Respir. Med. 2020, 176, 106272. [CrossRef]

14. Shah, A.S.; Wong, A.W.; Hague, C.J.; Murphy, D.T.; Johnston, J.C.; Ryerson, C.J.; Carlsten, C. A prospective study of 12-week respiratory outcomes in COVID-19-related hospitalisations. Thorax 2020. [CrossRef]

15. Daher, A.; Balfanz, P.; Cornelissen, C.; Müller, A.; Bergs, I.; Marx, N.; Müller-Wieland, D.; Hartmann, B.; Dreher, M.; Müller, T. Follow up of patients with severe coronavirus disease 2019 (COVID-19): Pulmonary and extrapulmonary disease sequelae. Respir. Med. 2020, 174, 106197. [CrossRef] [PubMed]

16. Mo, X.; Jian, W.; Su, Z.; Chen, M.; Peng, H.; Peng, P.; Lei, C.; Chen, R.; Zhong, N.; Li, S. Abnormal pulmonary function in COVID-19 patients at time of hospital discharge. Eur. Respir. J. 2020, 55, 2001217. [CrossRef]

17. Hsu, H.; Tzao, C.; Wu, C.; Chang, W.; Tsai, C.; Tung, H.; Chen, C. Correlation of high-resolution CT, symptoms, and pulmonary function in patients during recovery from severe acute respiratory syndrome. Chest 2004, 126, 149-158. [CrossRef]

18. Hui, D.S.; Wong, K.T.; Ko, F.W.; Tam, L.S.; Chan, D.P.; Woo, J.; Sung, J.J.Y. The 1-year impact of severe acute respiratory syndrome on pulmonary function, exercise capacity, and quality of life in a cohort of survivors. Chest 2005, 128, 2247-2261. [CrossRef]

19. Ahmed, H.; Patel, K.; Greenwood, D.C.; Halpin, S.; Lewthwaite, P.; Salawu, A.; Eyre, L.; Breen, A.; O'Connor, R.; Jones, A.; et al. Long-term clinical outcomes in survivors of severe acute respiratory syndrome and Middle East respiratory syndrome coronavirus outbreaks after hospitalisation or ICU admission: A systematic review and meta-analysis. J. Rehabil. Med. 2020, 52, 63. [CrossRef]

20. European Respiratory Society (ERS). Recommendation from ERS Group 9.1 (Respiratory Function Technologists/Scientists) Lung Function Testing during COVID-19 Pandemic and beyond 2020. Available online: https://ers.app.box.com/s/zs1uu88wy5 1monr0ewd990itoz4tsn $2 \mathrm{~h}$ (accessed on 6 December 2020).

21. Milanese, M.; Corsico, A.G.; Bellofiore, S.; Carrozzi, L.; Di Marco, F.; Iovene, B.; Richeldi, L.; Sanna, A.; Santus, P.; Schisano, M.; et al. Esami di Funzionalità Respiratoria nel Contesto COVID-19. Version 1.0. Italian Respiratory Society/Società Italiana di Pneumologia (IRS/SIP) 2020. Available online: https://irn.sipirs.it/storage/61/Documento-EsamiFunzionalit\%C3\%A0ResCovid_Vers.1_12.05.2020.pdf (accessed on 2 December 2020).

22. Santus, P.; Radovanovic, D.; Saderi, L.; Marino, P.; Cogliati, C.; De Filippis, G.; Rizzi, M.; Franceschi, E.; Pini, S.; Giuliani, F.; et al. Severity of respiratory failure at admission and in-hospital mortality in patients with COVID-19: A prospective observational multicentre study. BMJ Open 2020, 10, e043651. [CrossRef]

23. Aliberti, S.; Radovanovic, D.; Billi, F.; Sotgiu, G.; Costanzo, M.; Pilocane, T.; Saderi, L.; Gramegna, A.; Rovellini, A.; Perotto, L.; et al. Helmet CPAP treatment in patients with COVID-19 pneumonia: A multicentre cohort study. Eur. Respir. J. 2020, 56, 2001935. [CrossRef] [PubMed]

24. Wanger, J.; Clausen, J.L.; Coates, A.; Pedersen, O.F.; Brusasco, V.; Burgos, F.; Casaburi, R.; Crapo, R.; Enright, P.; van der Grinten, C.P.; et al. Standardisation of the measurement of lung volumes. Eur. Respir. J. 2005, 26, 511-522. [CrossRef] [PubMed]

25. Graham, B.L.; Brusasco, V.; Burgos, F.; Cooper, B.G.; Jensen, R.; Kendrick, A.; MacIntyre, N.R.; Thompson, B.R.; Wanger, J. 2017 ERS/ATS standards for single-breath carbon monoxide uptake in the lung. Eur Respir J 2017, 49, 1600016. [CrossRef]

26. Wasilewski, P.G.; Mruk, B.; Mazur, S.; Półtorak-Szymczak, G.; Sklinda, K.; Walecki, J. COVID-19 severity scoring systems in radiological imaging-A review. Pol. J. Radiol. 2020, 85, e361-e368. [CrossRef] [PubMed]

27. Li, K.; Fang, Y.; Li, W.; Pan, C.; Qin, P.; Zhong, Y.; Liu, X.; Liao, Y.; Li, S. CT image visual quantitative evaluation and clinical classification of coronavirus disease (COVID-19). Eur. Radiol. 2020, 30, 4407-4416. [CrossRef]

28. Radovanovic, D.; Rizzi, M.; Pini, S.; Saad, M.; Chiumello, D.A.; Santus, P. Helmet CPAP to treat acute hypoxemic respiratory failure in patients with COVID-19: A management strategy proposal. J. Clin. Med. 2020, 9, 1191. [CrossRef]

29. Vitacca, M.; Nava, S.; Santus, P.; Harari, S. Early consensus management for non-ICU acute respiratory failure SARS-CoV- 2 emergency in Italy: From ward to trenches. Eur. Respir. J. 2020, 55, 2000632. [CrossRef]

30. Bassetti, M.; Giacobbe, D.R.; Aliberti, S.; Barisione, E.; Centanni, S.; De Rosa, F.G.; Di Marco, F.; Gori, A.; Granata, G.; Mikulska, M.; et al. Italian Society of Anti-infective Therapy (SITA) and the Italian Society of Pulmonology (SIP). Balancing evidence and frontline experience in the early phases of the COVID-19 pandemic: Current position of the Italian Society of anti-infective therapy (SITA) and the Italian Society of pulmonology (SIP). Clin. Microbiol. Infect. 2020, 26, 880-894.

31. SIMIT (Società Italiana di Malattie Infettive e Tropicali). Sezione Regione Lombardia. In Vademecum per la Cura Delle Persone con Malattia da COVID-19, 2nd ed.; 2020; Available online: http:/ / www.simit.org/medias/1569-covid19-vademecum-13-03-202.pdf (accessed on 18 November 2020). 
32. Metlay, J.P.; Waterer, G.W.; Long, A.C.; Anzueto, A.; Brozek, J.; Crothers, K.; Cooley, L.A.; Dean, N.C.; Fine, M.J.; Flanders, S.A.; et al. Diagnosis and treatment of adults with community-acquired pneumonia. An official clinical practice guideline of the American Thoracic Society and Infectious Diseases Society of America. Am. J. Respir. Crit. Care Med. 2019, 200, e45-e67. [CrossRef]

33. Quanjer, P.H. Standardized Lung Function Testing: Report Working Party Standardization of Lung Function Tests; European Community for Coal and Steel: Luxembourg, 1983.

34. Wang, Y.; Dong, C.; Hu, Y.; Li, C.; Ren, Q.; Zhang, X.; Shi, H.; Zhou, M. Temporal changes of CT findings in 90 patients with COVID-19 pneumonia: A longitudinal study. Radiology 2020, 296, E55-E64. [CrossRef]

35. Paliogiannis, P.; Mangoni, A.A.; Dettori, P.; Nasrallah, G.K.; Pintus, G.; Zinellu, A. D-Dimer Concentrations and COVID-19 severity: A systematic review and meta-analysis. Front. Public Health 2020, 8, 432. [CrossRef]

36. Grasselli, G.; Tonetti, T.; Protti, A.; Langer, T.; Girardis, M.; Bellani, G.; Laffey, J.; Carrafiello, G.; Carsana, L.; Rizzuto, C.; et al. Pathophysiology of COVID-19-associated acute respiratory distress syndrome: A multicentre prospective observational study. Lancet Respir. Med. 2020, 8, 1201-1208. [CrossRef]

37. Hughes, J.M.B.; Pride, N.B. Examination of the carbon monoxide diffusing capacity (DL(CO)) in relation to its KCO and VA components. Am. J. Respir. Crit. Care Med. 2012, 186, 132-139. [CrossRef] [PubMed]

38. Johnson, D.C. Importance of adjusting carbon monoxide diffusing capacity (DLco) and carbon monoxide transfer coefficient (KCO) for alveolar volume. Respir. Med. 2000, 94, 28-37. [CrossRef] [PubMed] 